\title{
Agregação familiar na adiposidade do tronco: um estudo em famílias nucleares portuguesas
}

CDD. 20.ed. 614.4

616.042

\begin{tabular}{r|l} 
Michele SOUZA* & *Faculdade de Des- \\
Raquel CHAVES* & porto, Universidade do \\
Daniel SANTOS* & Porto - Portugal. \\
**nniversidade Federal \\
Rogério FERMINO* & do Paraná e Pontifícia \\
Rui GARGANTA* & Universidade Católica \\
do Paraná. \\
André SEABRA* & \\
J osé MAIA* &
\end{tabular}

\section{Resumo}

Este estudo teve como objetivos: 1) identificar a presença indireta de transmissão vertical de fatores genéticos entre progenitores e descendentes em dois fenótipos da adiposidade do tronco (AT); 2) estimar a contribuição dos fatores genéticos e ambientais responsáveis pela variabilidade fenotípica da adiposidade do tronco relativa ( $\mathrm{AT}_{\text {rel }}$ ) e absoluta $\left(\mathrm{AT}_{\text {abs }}\right.$ ) em termos populacionais. A amostra foi constituída por 422 indivíduos pertencentes a 107 famílias nucleares portuguesas. Os fenótipos da AT foram avaliados com um aparelho de BIA da marca Tanita ${ }^{\circledR}$ modelo BC- 418MA. A estrutura familiar e a análise do comportamento genérico das variáveis entre diferentes membros familiares foram realizadas no "software" PEDSTATS. Para calcular a correlação entre familiares foi utilizado o módulo FCOR do "software" de Epidemiologia Genética S.A.G.E 5.3. As estimativas de heritabilidade $\left(h^{2}\right)$ foram realizadas através do método de verossimilhança implementado no "software" SOLAR. Os valores dos coeficientes de correlação entre os diferentes graus de parentesco foram baixos a moderados para $A T_{\text {rel }}(0,205<r<0,738)$ e $\mathrm{AT}_{\text {abs }}(0,199<r<0,782)$. Os fatores genéticos explicaram 50 e $47 \%$ da variação dos fenótipos da $A T_{\text {rel }}$

e $\mathrm{AT}_{\text {abs' }}^{\text {abs }}$ respectivamente. Esses resultados: 1) indicam uma forte agregação familiar na AT de famílias nucleares portuguesas; 2 ) contribuem para estudos avançados de Epidemiologia Genética e 3 ) ressaltam a necessidade da implementação de intervenções físicas e nutricionais direcionadas a toda família.

UnItERMOS: Agregação familiar; Heritabilidade; Adiposidade do tronco; Epidemiologia genética.

\section{Introdução}

Um padrão marcadamente andróide ou distribuição central de tecido adiposo representa um problema clínico sério com implicações na saúde pública por induzir riscos diferenciados na manifestação de doenças crônicas, quando comparado com outras formas de deposição de gordura corporal (Davey, Ramachandran, Snehalatha, Hitman \& McKeigue, 2000; KatzmarzyK \& Bouchard, 2005; Pérusse, Despres, Lemieux, Rice, Rao \& Bouchard, 1996; Rice \& BoreCKI, 2001; WaLley, BlaKEMORE \& Froguel, 2006). Por exemplo, indivíduos com maior adiposidade do tronco (AT) têm maior predisposição para desenvolver doenças cardiovasculares e distúrbios metabólicos (Fermino, Seabra, Garganta, Valdivia \& Maia, 2008; Pérusse, Rice, Province, Gagnon, Leon, SkinNer, Wilmore, Rao \& Bouchard, 2000; Pérusse et al., 1996; Rice, Despres, Daw, Gagnon, Borecki, Pérusse, Leon, Skinner, Wilmore, Rao \& Bouchard, 1997; Wu, Heshka, Wang, Pierson Junior, Heymsfield, Laferrere, Wang, Albu, PiSUNYER \& GALLAGHER, 2007). Estudos populacionais sugerem que a AT apresenta, atualmente, elevada prevalência em diferentes estratos etários e tende a aumentar com a idade (Shen, PunYanitya, Silva, Chen, Gallagher, Sardinha, Allison \& HeYMSFIELD, 2009; Wu et al., 2007), acarretando um 
elevado impacto financeiro para a sociedade, devido aos altos custos (diretos e indiretos) gerados por essa situação de morbilidade (KATZMARZYK \& JANSSEN, 2004; Leal, Luengo-Fernández, Gray, Petersen \& Rayner, 2006; Lloyd-Jones, AdAms, Carnethon, De Simone, Ferguson, Flegal, Ford, Fuee, Go, GreENLUnd, HaAse et al., 2009).

A interação entre o ambiente e a herança genética, aliada ao estilo de vida sedentário, tem implicações importantes na explicação da variabilidade interindividual do acúmulo de gordura corporal central (BELL, Walley \& Froguel, 2005; Marti, Moreno-Aliaga, Hebebrand \& Martinez, 2004). Nos últimos anos, estudos no domínio da Epidemiologia Genética têm indicado que alguns indivíduos são mais suscetíveis a mudanças no padrão de distribuição de AT, sugerindo que os fatores genéticos desempenham uma função relevante na etiologia desse fenótipo poligênico e multifatorial (FERMino et al., 2008). Pesquisas com delineamento familiar mostraram que tanto os genes como o ambiente comumente partilhado contribuem para a agregação familiar $(\mathrm{AgF})$ na adiposidade relativa $\left(\mathrm{AT}_{\text {rel }}\right)$ e absoluta do tronco $\left(\mathrm{AT}_{\mathrm{abs}}\right.$ ), estimando que cerca de 31 (Butte, CaI, Cole \& Comuzzie, 2006; Butte, Comuzzie, Cole, Mehta, Cai, Tejero, Bastarrachea \& Smith, 2005) a $64 \%$ (Hsu, LeNCHIK, NiCKLAs, LoHMAN, RegisTer, Mychaleckyj, Langefeld, Freedman, Bowden \& CARR, 2005) da variância fenotípica total é devido à influência dos fatores genéticos.

A estimativa precisa da quantidade de gordura central tem sido efetuada a partir de técnicas, indiretas e duplamente indiretas, que variam em termos de validade, fidedignidade e objetividade (Monteiro \& Fernandes Filho, 2002). Para a AT, os indicadores mais utilizados em pesquisa epidemiológica de larga escala amostral são o perímetro

\section{Métodos}

\section{Amostra}

Este estudo éde natureza transversal e sua amostragem foi efetuada com base no contato com escolas de diferentes distritos da região norte de Portugal. Foi enviada uma comunicação escrita a cada família convidando-a a participar no estudo. Todos assinaram o consentimento livre e informado e foram avaliados no período matutino e em jejum. A amostra foi constituída por 107 famílias nucleares (duas gerações), totalizando da cintura e as pregas de adiposidade subcutânea. Mais recentemente, a impedância bioelétrica (BIA) passou a ser um instrumento útil devido ao fato de fornecer informação segmentar do corpo, além de ser relativamente mais barata que os métodos laboratoriais, como a ressonância magnética nuclear, tomografia computadorizada (TC) e densitometria computadorizada por absorciometria radiológica de dupla energia (DXA). Além disso, a BIA não é um método invasivo, tem fácil aplicação e portabilidade (Jaffrin \& Morel, 2009; Monteiro \& Fernandes FiLHO, 2002), a que se associa validade concorrente bem estabelecida, quando comparada à DXA (Pietrobelli, Rubiano, St-Onge \& HeYMSField, 2004).

Diversas pesquisas procuraram quantificar a influência dos fatores genéticos na AT, sendo que a maioria foi realizada na população americana (BUTTE et al., 2005, 2006; Comuzzie, Blangero, Mahaney, Mitchell, Stern \& MacCluer, 1993; Hsu et al., 2005; $\mathrm{CHOH}$, Demerath, Lee, Williams, Towne, Siervogel, Cole \& CZERWINSKI, 2009), a partir de informação obtida com o DXA (ButTe et al., 2005, 2006; СHOH et al., 2009; Hsu et al., 2005) e medidas antropométricas (ComuzZie et al., 1993; Davey et al., 2000; PÉrusse et al., 2000). O único estudo de Epidemiologia Genética em língua portuguesa, que conseguimos localizar, foi realizado por FERMINO et al. (2008) cujos fenótipos da composição corporal foram medidos com um aparelho de BIA. Não é de nosso conhecimento que no espaço lusófono haja estudos de Epidemiologia Genética sobre a AT. Daqui que os objetivos da presente pesquisa sejam os seguintes: 1) identificar a presença indireta de transmissão vertical de fatores genéticos entre progenitores e descendentes nos dois fenótipos da AT; 2) estimar a contribuição dos fatores genéticos e ambientais responsáveis pela variabilidade fenotípica da $\mathrm{AT}_{\mathrm{rel}}$ e $\mathrm{AT}_{\mathrm{abs}}$, em termos populacionais.
422 indivíduos (214 progenitores - 40,9 \pm 4,5 anos e 208 descendentes - 13,2 \pm 3 anos); no entanto, face a problemas de informação omissa, o número total de sujeitos analisados foi de 381 (TABELA 1).

\section{Procedimentos}

Os fenótipos da AT foram avaliados com um aparelho de BIA da marca Tanita ${ }^{\circledR}$ modelo BC-418MA (Tanita Corp., Tokyo, Japan). Esse instrumento 
realiza uma mensuração segmentar, fornecendo informaçōes sobre cinco partes corporais (membros inferiores, membros superiores e tronco) por meio de oito eletrodos (quatro nas mãos e quatro nos pés). Do seu "output" constam valores corporais totais e segmentares da adiposidade relativa, absoluta, massa isenta de gordura, bem como outras variáveis. A estimativa da gordura absoluta do tronco tem em consideração a gordura total do corpo, enquanto a estimativa da gordura relativa é independente do total.

Foi também medida a altura, o peso e calculado o índice de massa corporal (IMC). A altura foi medida com um antropômetro portátil da marca Siber Hegner®, com precisão de $0,1 \mathrm{~cm}$, segundo as referências de Lohman, Roche e Martorell (1988). O peso foi mensurado no mesmo aparelho de BIA, que possui uma precisão de $0,1 \mathrm{~kg}$. $\mathrm{O}$ indivíduo deveria estar na posição antropométrica de referência, vestindo roupas leves e descalço (LoHman, Roche \& Martorell, 1988). O IMC foi calculado pela razão entre a massa corporal e a altura ao quadrado, obtendo um valor final expresso em $\mathrm{kg} / \mathrm{m}^{2}$.

\section{Análise estatística}

A análise exploratória dos dados foi realizada no "software" SPSS 17.0, com o propósito de verificar possíveis erros de entrada dos dados, presença de "outliers", normalidade das distribuiçôes, bem como para calcular médias, desvios-padrão e amplitudes de variação. A verificação da estrutura familiar, bem como a análise do comportamento genérico das variáveis entre os diferentes membros familiares foram realizadas no "software" Pedstats. Para identificar a $\mathrm{AgF}$, através dos coeficientes de correlação, foi utilizado o módulo FCOR do "software" de Epidemiologia Genética S.A.G.E 5.3. As estimativas de heritabilidade $\left(\mathrm{h}^{2}\right)$ foram obtidas no "software SOLAR 4.0, através do método de máxima verossimilhança. Primeiramente, é formulado um modelo esporádico em que se assume uma ausência de covariância entre familiares para os fenótipos avaliados. Um segundo modelo é estabelecido, designado de poligênico. Os valores de ajustamento desses modelos (-2 "Loglikelihood") são contrastados e suas diferenças apresentam uma distribuição aproximada ao qui-quadrado com 1 grau de liberdade. Esperase que o valor do -2 "Loglikelihood" do segundo modelo (poligênico) seja menor que do primeiro e que o resultado da estatística do qui-quadrado seja significativo. Os dois fenótipos da AT foram ajustados às covariáveis sexo, idade, sexo*idade, idade $^{2}$, sexo*idade e IMC. Foi adotado o nível de significância de 0,05.

\section{Resultados}

A TABELA 1 apresenta a estatística descritiva das características dos sujeitos da amostra. Em média, os progenitores apresentaram valores de IMC semelhantes, situados na zona do sobrepeso (IMC > $25 \mathrm{~kg} /$ $\mathrm{m}^{2}$ ). Entre os descendentes, os valores médios de IMC revelam que os filhos e filhas são normoponderais para a sua idade (Cole, Bellizzi, Flegal \& Dietz, 2000). $\mathrm{Na}$ gordura corporal (GC), relativa e absoluta, as mães apresentam valores médios maiores que os pais, bem como, as filhas maiores que os filhos. $\mathrm{Na} \mathrm{AT}_{\text {rel }}$ as mães tiveram valores médios mais elevados que os pais, 28,9 e 23,7\%, respectivamente. Esse dimorfismo também foi verificado entre os descendentes, onde as meninas apresentaram, em média, $5 \%$ a mais de AT que os meninos. $\mathrm{Na} \mathrm{AT}_{\mathrm{abs}}$, os progenitores tiveram valores médios semelhantes; as filhas acumulam mais quilogramas de gordura no tronco do que os filhos.

As correlações entre os familiares, para os fenótipos da AT, estão na TABELA 2. Entre os cônjugues, o valor do coeficiente de correlação $(r)$ da $A_{\text {abs }}$ foi moderado $(r=0,376)$ e estatisticamente significativo. $\mathrm{Na}$ relação entre progenitores e descendentes, as correlações para $\mathrm{AT}_{\text {rel }}$ situaram-se entre 0,205 e 0,485 , sendo o valor mais expressivo encontrado na relação pai-filho. Para a $\mathrm{AT}_{\mathrm{abs}}$ todas as correlações foram positivas; as relações mãe-filho $(r=0,445)$ e pai-filho $(r=0,688)$ foram as que apresentaram valores mais altos. Entre os descendentes, as correlações entre irmão-irmão foram elevadas tanto para $\mathrm{AT}_{\text {rel }}$ como $\mathrm{AT}_{\mathrm{abs}}(0,738$ e 0,782$)$, respectivamente. 
TABELA 1 - Medidas descritivas das variáveis da amostra.

IMC: índice de massa corporal;

$\mathrm{GC}_{\text {rel }}$; gordura corporal relativa;

$\mathrm{GC}_{\mathrm{abs}}$ : gordura corporal absoluta;

$\mathrm{AT}_{\mathrm{rel}}$ : adiposidade do tronco relativa;

$\mathrm{AT}_{\text {abs }}$ : adiposidade do tronco absoluta;

m: média;

dp: desvio-padrão.

$A T_{\text {rel }}:$ adiposidade do tronco relativa;

$\mathrm{AT}_{\mathrm{abs}}$ : adiposidade do tronco absoluta;

$r$ : coeficiente de correlação;

ep: erro padrão

${ }^{*} p<0,05$.
Variáveis

\begin{tabular}{l} 
Idade (anos) \\
Massa corporal $(\mathrm{kg})$ \\
Altura $(\mathrm{cm})$ \\
$\mathrm{IMC}\left(\mathrm{kg} / \mathrm{m}^{2}\right)$ \\
$\mathrm{GC}_{\mathrm{rel}}(\%)$ \\
$\mathrm{GC}_{\mathrm{abs}}(\mathrm{kg})$ \\
$\mathrm{AT}_{\mathrm{rel}}(\%)$ \\
$\mathrm{AT}_{\text {abs }}(\mathrm{kg})$ \\
\hline
\end{tabular}

Pais

\begin{tabular}{ccc}
\hline $\mathbf{n}$ & $\mathbf{m} \pm \mathbf{d p}$ & amplitude \\
\hline 72 & $42,5 \pm 4,1$ & $33-56$ \\
64 & $77,1 \pm 11,4$ & $52,8-119,2$ \\
64 & $168,2 \pm 5,6$ & $155,6-185,5$ \\
64 & $27,4 \pm 3,7$ & $18,7-42,6$ \\
64 & $22,2 \pm 5$ & $8-39$ \\
64 & $17 \pm 6,3$ & $4,3-46,7$ \\
64 & $23,7 \pm 5,5$ & $6,6-40,2$ \\
64 & $10,3 \pm 3,5$ & $2-25$
\end{tabular}

\section{Progenitores}

\begin{tabular}{ccc}
\multicolumn{3}{c}{ Mães } \\
\hline $\mathbf{n}$ & $\mathbf{m} \pm \mathbf{d p}$ & amplitude \\
\hline 103 & $39,9 \pm 4,4$ & $30-53$ \\
100 & $68,4 \pm 11,7$ & $47,2-103$ \\
100 & $156,9 \pm 5,2$ & $144,2-171,6$ \\
100 & $27,8 \pm 4,9$ & $20-43,4$ \\
97 & $33,7 \pm 6,5$ & $18-51$ \\
98 & $23,7 \pm 8,4$ & $9,3-52$ \\
98 & $28,9 \pm 7,3$ & $11,7-47,1$ \\
98 & $10,9 \pm 4,2$ & $3,3-25$ \\
\hline
\end{tabular}

\section{Descendentes}

Idade (anos)

Massa corporal $(\mathrm{kg})$

Altura $(\mathrm{cm})$

IMC $\left(\mathrm{kg} / \mathrm{m}^{2}\right)$

$\mathrm{GC}_{\text {rel }}(\%)$

$\mathrm{GC}_{\text {abs }}(\mathrm{kg})$

$\mathrm{AT}_{\text {rel }}(\%)$

$\mathrm{AT}_{\text {abs }}(\mathrm{kg})$
Pais

\begin{tabular}{ccc}
\multicolumn{3}{c}{ Pais } \\
\hline $\mathbf{n}$ & $\mathbf{m} \pm \mathbf{d p}$ & amplitude \\
\hline 83 & $12,9 \pm 2,7$ & $7-19$ \\
81 & $50,7 \pm 18,2$ & $20,3-114,7$ \\
81 & $155,3 \pm 15,7$ & $104,4-181$ \\
81 & $20,4 \pm 4,2$ & $12,6-38,5$ \\
81 & $19,1 \pm 5,8$ & $12-42$ \\
81 & $10,1 \pm 6,6$ & $2,7-48,1$ \\
81 & $14,7 \pm 5,9$ & $6,5-30,1$ \\
81 & $4,3 \pm 3$ & $0,9-21,2$
\end{tabular}

\begin{tabular}{ccc}
\multicolumn{3}{c}{ Mães } \\
\hline $\mathbf{n}$ & $\mathbf{m} \pm \mathbf{d p}$ & amplitude \\
\hline 123 & $13,5 \pm 3,2$ & $7-25$ \\
122 & $50,4 \pm 12,3$ & $22,9-92$ \\
122 & $154,1 \pm 9,6$ & $119-169,5$ \\
122 & $21 \pm 3,8$ & $13,8-35,8$ \\
122 & $26 \pm 5,4$ & $17-45$ \\
122 & $13,6 \pm 5,9$ & $4,3-41,6$ \\
121 & $19,8 \pm 6,1$ & $10-43,3$ \\
121 & $5,7 \pm 3$ & $1,60-21,30$
\end{tabular}

TABELA 2 - Coeficientes de correlação (intraclasse entre elementos do mesmo sexo e interclasse nos de sexo oposto) entre familiares para a adiposidade relativa e absoluta do tronco.

\begin{tabular}{lccc}
\hline \multicolumn{1}{c}{ Grau de parentesco } & número de pares & $\begin{array}{c}\mathbf{A T}_{\text {rel }} \\
\mathbf{r} \pm \mathbf{e p}\end{array}$ & $\begin{array}{c}\mathbf{A T}_{\text {abs }} \\
\mathbf{r} \pm \mathbf{e p}\end{array}$ \\
\hline Cônjuges & 58 & $0,176 \pm 0,190$ & $0,376 \pm 0,115^{*}$ \\
Pai-Filho & 53 & $0,485 \pm 0,117^{*}$ & $0,688 \pm 0,080^{*}$ \\
Pai-Filha & 75 & $-0,001 \pm 0,993$ & $0,050 \pm 0,664$ \\
Mãe-Filho & 74 & $0,202 \pm 0,124$ & $0,445 \pm 0,103^{*}$ \\
Mãe-Filha & 116 & $0,205 \pm 0,093^{*}$ & $0,199 \pm 0,088^{*}$ \\
Irmão-Irmão & 11 & $0,738 \pm 0,143^{*}$ & $0,782 \pm 0,122^{*}$ \\
Irmã-Irmã & 34 & $0,141 \pm 0,172$ & $-0,051 \pm 0,173$ \\
Irmão-Irmã & 59 & $0,013 \pm 0,134$ & $0,075 \pm 0,131$ \\
\hline
\end{tabular}

As estimativas de $\mathrm{h}^{2}$ foram moderadas e estatisticamente significativas para os dois fenótipos da AT $\left(\mathrm{AT}_{\mathrm{rel}}=0,50\right.$ e $\left.\mathrm{AT}_{\text {abs }}=0,47\right)$. As covariáveis explicaram 48 e $56 \%$ da variância total da $\mathrm{AT}_{\mathrm{rel}}$ e $\mathrm{AT}_{\mathrm{abs}}$, respectivamente (TABELA 3).

156 • Rev. bras. Educ. Fís. Esporte, São Paulo, v.25, n.1, p.153-61, jan./mar. 2011 
TABELA 3 - Resultados dos testes dos modelos para os fenótipos da AT, valores do qui-quadrado obtidos pelas hipóteses formuladas e estimativas de heritabilidade $h^{2}$ ( \pm erro-padrão) ajustadas para covariáveis.

\begin{tabular}{cccccc}
\hline \multirow{2}{*}{ Fenótipo } & \multicolumn{2}{c}{ Modelos } & \multirow{2}{*}{$\chi^{2}(\mathbf{g l})$} & $\mathbf{h}^{2} \pm \mathbf{e p}$ & $\begin{array}{c}\text { Variância explicada } \\
\text { pelas covariáveis (\%) }\end{array}$ \\
\cline { 2 - 3 } & $\mathbf{A}$ & $\mathbf{B}$ & & & \\
\hline $\mathrm{AT}_{\mathrm{rel}}$ & $-972,25$ & $-960,37$ & $23,77(1)^{*}$ & $0,50 \pm 0,10^{*}$ & $48 \%^{\mathrm{a}}$ \\
$\mathrm{AT}_{\mathrm{abs}}$ & $-725,40$ & $-715,44$ & $19,91(1)^{*}$ & $0,47 \pm 0,10^{*}$ & $56 \%^{\mathrm{b}}$ \\
\hline
\end{tabular}

\section{Discussão}

O propósito fundamental deste estudo foi identificar a presença indireta de transmissão vertical de fatores genéticos e estimar a contribuição desses fatores, bem como dos ambientais, na variabilidade da $\mathrm{AT}_{\mathrm{rel}}$ e $\mathrm{AT}_{\mathrm{abs}}$ medidos com um aparelho de BIA. Como mencionado anteriormente, optamos por este método por sua fácil aplicação e portabilidade, bem como validade concorrente bem estabelecida com o DXA, que embora seja um método "gold standard" na avaliação da composição corporal, também apresenta alguns fatores limitantes, resultantes das suas equações e pressupostos de avaliação que diferem entre os fabricantes e "software" utilizado para a análise (BROWNBILL \& Ilich, 2005; Helba \& BinKOVITZ, 2009).

De maneira geral, os resultados obtidos da GC e do IMC dos progenitores e descendentes foram semelhantes aos reportados em pesquisas prévias (DAveY et al., 2000; ButTe et al., 2005, 2006; FERMinO et al., 2008; Hsu et al., 2005). Para a AT rel, indivíduos do sexo feminino, de ambas as geraçóes, apresentaram maior porcentagem de gordura, tal como sugerido anteriormente no estudo com 244 famílias nucleares americanas (Hsu et al., 2005), mas não corroboradas na pesquisa canadense de Pérusse et al. (2000). Não foram encontradas investigações sobre a $\mathrm{AT}_{\text {abs }}$. É importante salientar que ao contrastar as médias da $\mathrm{GC}_{\mathrm{abs}} \operatorname{com} \mathrm{AT}_{\mathrm{abs}}$ dos progenitores, os pais demonstraram tendência ao padrão andróide de distribuição de gordura, e as mães ao padrão ginóide. Sendo assim, eles podem tornarse mais suscetíveis ao desenvolvimento de distúrbios metabólicos, bem como à manifestação de doenças crônicas, sobretudo as de origem cardiovascular (KaTZMARZYK \& BOUCHARD, 2005; Rice \& BORECKI, 2001; Walley, Blakemore \& Froguel, 2006).

As pesquisas de $\mathrm{AgF}$ da AT procuram quantificar a magnitude da influência dos fatores genéticos na variação populacional num dado fenótipo. A estatística mais simples para estimar essa semelhança familiar é a correlação (r). O presente estudo mostra valores significativos entre 0,205 e 0,738 para $\mathrm{AT}_{\text {rel }} ; 0,199 \mathrm{e}$ 0,782 para $\mathrm{AT}_{\text {abs }}$, nas oito correlações calculadas no seio familiar. Esses valores sugerem a presença de $\mathrm{AgF}$ na AT, embora com um padrão distinto de semelhança entre pares. A maior correlação foi verificada entre os irmãos, tanto para $\mathrm{AT}_{\text {rel }}$ como $\mathrm{AT}_{\text {abs }}$, sugerindo influência clara dos fatores genéticos nesses traços. Os resultados obtidos entre os cônjuges foram baixos a moderados e expressam a importância do ambiente comumente partilhado, uma vez que não existe qualquer partilha de material genético entre eles.

PÉRUsSe et al. (2000) analisaram 99 famílias nucleares participantes do "HERITAGE Family Study" e, de forma geral, encontraram valores de $\mathrm{r}$ mais baixos para $\mathrm{AT}_{\text {rel }}(\mathrm{r}=0,19-0,21)$, quando comparados com a presente pesquisa. No mesmo sentido, DAVEY et al. (2000) estudaram 1295 indivíduos indianos pertencentes a 300 famílias nucleares e verificaram correlações entre progenitores $(r=0,29)$, progenitoresdescendentes $(r=0,20)$ e descendentes $(r=0,46)$, para $\mathrm{AT}_{\mathrm{rel}}$. $\mathrm{O}$ valor reportado entre progenitores e descendentes foi semelhante ao encontrado na relação mãe-filho $(r=0,20)$, mas inferior à relação pai filho $(r$ $=0,48)$ desta pesquisa. Em Portugal, um estudo com famílias nucleres de FERMINOet al. (2008) identificaram AgF nos diferentes fenótipos da composição corporal; as correlaçóes positivas mais altas, para $\mathrm{AT}_{\text {rel}}$, foram entre pai-filho $(r=0,20)$, irmã-irmã $(r=0,22)$ e mãe-filha $(r=0,23)$, apresentando semelhança com o presente estudo apenas na relação mãe-filha.

$\mathrm{O}$ passo seguinte da $\mathrm{AgF}$ é estimar a magnitude dos efeitos genéticos $\left(h^{2}\right)$ a governar a variação encontrada nos fenótipos em apreço, que pode ser realizado pelo método de máxima verossimilhança (no pressuposto do fenótipo ter uma distribuição normal multivariada), ou pelo método de "Generalized Estimating Equations”. Como a distribuição da AT é multinormal, as estimativas de $\mathrm{h}^{2}$ são precisas face à elevada eficiência da máxima verossimilhança.
A: valor do -2 "loglikelihood" do modelo esporádio (variância residual);

B: Valor do -2 "loglikelihood" do modelo poligênico (efeitos aditivos genéticos);

$\chi^{2}$ : qui-quadrado; gl: grau de liberdade; $\mathrm{h}^{2}$ : estimativa de heritabilidade; ep: erro padrão. AT : Adiposidade do tronco relativa; $\mathrm{AT}_{\text {abs }}$ : adiposidade do tronco absoluta. Covariáveis ajustadas: asexo e idade: bidade e idade 2 ${ }^{*} p<0,001$. 
Os resultados deste estudo mostram que os fatores genéticos podem explicar 50 e $47 \%$ da variação fenotípica total da $\mathrm{AT}_{\mathrm{rel}}$ e $\mathrm{AT}_{\mathrm{abs}}$, respectivamente. Esses resultados indicam uma influência genética substancial nos fenótipos da obesidade central. Informações provenientes de investigações em Epidemiologia Genética são escassas na população portuguesa. Só tivemos acesso ao estudo de Fermino et al. (2008), citado anteriormente, em que se estimou $\mathrm{h}^{2}$ de $35 \%$ para $\mathrm{AT}_{\text {rel }}$ numa amostra semelhante a presente pesquisa. Estudos em outras populações apresentam, de modo geral, $\mathrm{h}^{2}$ baixa a moderada para $\mathrm{AT}_{\text {rel }}$ não havendo informações disponíveis sobre a $\mathrm{AT}_{\text {abs }}$. ButTE et al. $(2005,2006)$ realizaram duas pesquisas nos EUA, com amostras semelhantes pertencentes ao "Viva La Familia Study" e constataram que os fatores genéticos explicavam $31 \%$ dos valores da $\mathrm{AT}_{\text {rel }}$. Um estudo realizado na população americana (CoMUZZIE et al., 1993) e outro no Canadá (Pérusse et al., 2000) reportaram estimativas de $^{2}$ semelhantes, 0,38 e 0,36, respectivamente. Mais recentemente, $\mathrm{CHOH}$ et al. (2009) verificaram $\mathrm{AgF}$ em 521 indivíduos americanos, com idades entre 18 e 86 anos e reportaram $\mathrm{h}^{2}$ de $35 \%$ para $\mathrm{AT}_{\text {rel }}$. Esse estudo também teve como objetivo averiguar a correlação genética (rg) entre fenótipos que marcam a obesidade central e atividade física esportiva. Para AT, essa correlação foi significativamente diferente de $-1(\mathrm{rg}=-0,36)$, indicando pleiotropia incompleta entre os dois traços, i.e., AT e atividade física esportiva são influenciadas, na mesma extensão, pelos mesmos genes; contudo, também apresentam bases genéticas únicas (DUREN, Sherwood, Czerwinski, Chumlea, Lee, Demerath, Sun, Siervogel \& Towne, 2008). Essa sugestão é importante, uma vez que induz a elaboração e aplicação de estratégias de intervenção de atividade física, visando uma maior e melhor sensibilização desses indivíduos, relativamente à prática esportiva. KAY e FiaTARONE SINGH (2006), através de uma revisão sistemática de 37 estudos, concluíram que o exercicío físico é a intervenção mais efetiva para a redução da AT e gordura visceral em sujeitos com sobrepeso e obesidade. Nesse mesmo sentido, uma pesquisa recente (HUNTER, BROCK, Byrne, Chandler-Laney, Del Corral \& Gower, 2009) com 97 mulheres constatou que entre essas, as que praticavam exercícios aeróbios e/ou de força não apresentaram, após um ano de acompanhamento, um aumento significativo de gordura central, principalmente visceral, quando comparadas às mulheres que não praticavam atividade física. Esses resultados sugerem que a suscetibilidade genética para a obesidade pode ser contornada, pelos menos em parte, pela adoção de um estilo de vida fisicamente ativo.
Todas as investigações supracitadas apresentaram estimativas de $\mathrm{h}^{2}$ inferior a do presente estudo, à exceção da pesquisa de $\mathrm{Hsu}$ et al. (2005), cuja $\mathrm{h}^{2}$ foi de $63 \%$ para $\mathrm{AT}_{\text {rel }}$. Essa variabilidade dos resultados pode ser explicada, em parte, pelas características distintas de cada estudo, como por exemplo, a dimensão da amostra, composição das famílias (nucleares ou "pedigrees" extensos), idade, aspectos étnicos e culturais, entre outros fatores do estudo. Contudo, ainda que sejam consideradas as diferentes características das pesquisas, há que ser cauteloso na comparação direta das estimativas de $\mathrm{h}^{2}$, dado ser uma estimativa específica de cada população e contexto ambiental (RICE \& BORECKI, 2001). No que concerne à composição das famílias, alguns autores $(\mathrm{CHOH}$ et al., 2009; Rice \& BORECKI, 2001) salientam que um delineamento com famílias nucleares não estima, de maneira isolada, a contribuição dos fatores genéticos e ambientais, e que um estudo com "pedigrees" extensos permite calcular essas duas quantidades.

A identificação de $\mathrm{AgF}$ na AT representa a base para a realização de estudos mais avançados no domínio da Genética Molecular. Através das fases III e IV da Epidemiologia Genética é possível identificar regiōes cromossômicas que possam conter genes candidatos, bem como mutações genéticas responsáveis pela variabilidade da AT. Não conseguimos localizar estudos de "linkage" para AT, somente para outros fenótipos da obesidade central, como a AV (Rankinen, Zuberi, Chagnon, Weisnagel, Argyropoulos, Walts, Pérusse \& BOUCHARD, 2006). Entretanto, um estudo recente verificou associação entre um "single-nucleotide polymorphism" (SNP) do gene FTO com o acúmulo de AT (Lopez-Bermejo, Petry, Diaz, Sebastiani, De Zegher, Dunger \& Ibanez, 2008). Ruth Loos (2009), em um estudo de revisão sobre o progresso da investigação genética em obesidade, também referencia associação entre variações desse mesmo gene com diferentes expressões da obesidade. A autora salienta que apesar dos muitos anos de pesquisa, somente recentemente foi alcançado um maior sucesso na compreensão mais profunda da fisiopatologia desse fenótipo altamente complexo. Entretanto, Ruth Loos acredita que a grande dificuldade das descobertas a nível molecular reside na tradução dos conhecimentos em termos de saúde pública, sobretudo no esclarecimento do risco genético individual.

O presente estudo apresenta alguns pontos fortes: 1) situar-se no domínio da Epidemiologia Genética aplicada à AT; 2) ter uma amostra 
proveniente de um país lusófono (onde há a escassez de investigaçóes semelhantes e por ser expectável a ocorrência de diferentes estimativas de efeitos genéticos); 3) utilizar um procedimento não invasivo e altamente fiável na estimação da AT (método de bioimpedância). Há, contudo, limitações que importa destacar: 1) ausência de consideração sobre os níveis de atividade física dos participantes da pesquisa; 2) hábitos nutricionais e, 3) a necessidade da presença de maior número de elementos em cada família (sentido vertical e horizontal) para estimar efeitos ambientais comuns.
Conclui-se que os resultados da presente pesquisa salientam a presença significativa de $\mathrm{AgF}$ nos fenótipos de AT de famílias nucleares portuguesas e contribuem para estudos futuros nas demais fases da Epidemiologia Genética. Ao mesmo tempo, sugerem a necessidade de implementação de propostas nutricionais e de atividade física dirigida a todos elementos da família. Nesses últimos, seria relevante pensar em estratégias de intervenções multifacetadas, de elevada adesão em atividades cujo dispêndio energético, moderado-a-elevado, fosse mais frequente e sistemático.

\begin{abstract}
Familial aggregation on trunkal fat: a study with Portuguese families

The aims of this study was to 1) identify the presence of indirect transmission of genetic factors between parents and children in two phenotypes of trunk fat, and 2) estimate the contribution of genetic and environmental factors responsible for phenotypic variation on relative trunk fat and absolute trunk fat. The sample consisted of 422 individuals from 107 Portuguese nuclear families. Trunk fat phenotypes were measured with a bioelectric impedance device Tanita ${ }^{\circledR}$ model BC-418MA. Family structure and analysis of the generic behavior of the variables between different family members were verified in PEDSTATS software. Familiar correlations were computed in the FCOR module of SAGE 5.3 software. Heritability estimates $\left(h^{2}\right)$ were performed using the likelihood method implemented in SOLAR software. Correlation coefficients between relatives were low to moderate on relative trunk fat $(0.205<r<0.738)$ and absolute trunk fat $(0.199<r<0.782)$. Genetic factors explained 50 and $47 \%$ of the variation in phenotypes of relative and absolute trunk fat, respectively. These results 1) indicate a strong familial aggregation on trunk fat of Portuguese nuclear families, 2) contribute to advanced studies in Genetic Epidemiology 3 ) and emphasize the need for physical and nutritional interventions directed to all family members.
\end{abstract}

UnITERMS: Familial aggregation; Heritability; Trunk fat; Genetic epidemiology.

\title{
Referências
}

BELL, C.G.; WALLEY, A.J.; FROGUEL, P. The genetics of human obesity. Nature Reviews: Genetics, London, v.6, n.3, p.221-34, 2005.

BROWNBILL, R.A.; ILICH, J.Z. Measuring body composition in overweight individuals by dual energy x-ray absorptiometry. BMC Medical Imaging, London, v.5, n.1, p.1, 2005.

BUTTE, N.F.; CAI, G.; COLE, S.A.; COMUZZIE, A.G. Viva la Familia Study: genetic and environmental contributions to childhood obesity and its comorbidities in the Hispanic population. American Journal of Clinical Nutrition, Bethesda, v.84, n.3, p.646-54, 2006.

BUTTE, N.F.; COMUZZIE, A.G.; COLE, S.A.; MEHTA, N.R.; CAI, G.; TEJERO, M.; BASTARRACHEA, R.; SMITH, E.O. Quantitative genetic analysis of the metabolic syndrome in Hispanic children. Pediatric Research, Hagerstown, v.58, n.6, p.1243-8, 2005.

CHOH, A.C.; DEMERATH, E.W.; LEE, M.; WILLIAMS, K.D.; TOWNE, B.; SIERVOGEL, R.M.; COLE, S.A.; CZERWINSKI, S.A. Genetic analysis of self-reported physical activity and adiposity: the Southwest Ohio Family Study. Public Health Nutrition, Wallingford, v.12, n.8, p.1052-60, 2009. 
COLE, T.J.; BELLIZZI, M.C.; FLEGAL, K.M.; DIETZ, W.H. Establishing a standard definition for child overweight and obesity worldwide: international survey. British Medical Journal, London, v.320, n.7244, p.1240-3, 2000.

COMUZZIE, A.G.; BLANGERO, J.; MAHANEY, M.C.; MITCHELL, B.D.; STERN, M.P.; MacCLUER, J.W. Quantitative genetics of sexual dimorphism in body fat measurements. American Journal of Human Biology, Inew York, v.5, p.725-34, 1993.

DAVEY, G.; RAMACHANDRAN, A.; SNEHALATHA, C.; HITMAN, G.A.; McKEIGUE, P.M. Familial aggregation of central obesity in Southern Indians. International Journal of Obesity and Related Metabolic Disorders, London, v.24, n.11, p.1523-7, 2000.

DUREN, D.L.; SHERWOOD, R.J.; CZERWINSKI, S.A.; CHUMLEA, W.C.; LEE, M.; DEMERATH, E.W.; SUN, S.S.; SIERVOGEL, R.M.; TOWNE, B. Genetic architecture of knee radiographic joint space in healthy young adults. Human Biology: an International Record of Research, Detroit, v.80, n.1, p.1-9, 2008.

FERMINO, R.C.; SEABRA, A.; GARGANTA, R.; VALDIVIA, A.B.; MAIA, J. Um estudo de genética quantitativa sobre agregação familiar na composição corporal de famílias nucleares portuguesas. Revista Portuguesa de Ciências do Desporto, Porto, v.8, n.1, p.77-84, 2008.

HELBA, M.; BINKOVITZ, L.A. Pediatric body composition analysis with dual-energy X-ray absorptiometry. Pediatric Radiology, Berlin, v.39, n.7, p.647-56, 2009.

HSU, F.C.; LENCHIK, L.; NICKLAS, B.J.; LOHMAN, K.; REGISTER, T.C.; MYCHALECKYJ, J.; LANGEFELD, C.D.; FREEDMAN, B.I.; BOWDEN, D.W.; CARR, J.J. Heritability of body composition measured by DXA in the diabetes heart study. Obesity Research, Silver Spring, v.13, n.2, p.312-9, 2005.

HUNTER, G.R.; BROCK, D.W.; BYRNE, N.M.; CHANDLER-LANEY, P.C.; DEL CORRAL, P.; GOWER, B.A. Exercise training prevents regain of visceral fat for 1 year following weight loss. Obesity, Silver Spring, v.18, n.4, p.690-5, 2009.

JAFFRIN, M.Y.; MOREL, H. Measurements of body composition in limbs and trunk using a eight contact eletrodes impedancemeter. Medical Engineering \& Physics, London, v.31, p.1079-86, 2009.

KATZMARZYK, P.T.; BOUCHARD, C. Genetic influences on human body composition. In: HEYMSFIELD, S.B.; LOHMAN, T.G.; WANG, Z. Human body composition. Champaign: Human Kinetics, 2005.

KATZMARZYK, P.T.; JANSSEN, I. The economic costs associated with physical inactivity and obesity in Canada: an update. Canadian Journal Applied Physiology, Champaign, v.29, n.1, p.90-115, 2004.

KAY, S.J.; FIATARONE SINGH, M.A. The influence of physical activity on abdominal fat: a systematic review of the literature. Obesity Reviews, Silver Spring, v.7, n.2, p.183-200, 2006.

LEAL, J.; LUENGO-FERNÁNDEZ, R.; GRAY, A.; PETERSEN, S.; RAYNER, M. Economic burden of cardiovascular disease in the enlarged European Union. European Heart Journal, Oxford, v.27,p.1610-9, 2006.

LLOYD-JONES, D.; ADAMS, R.; CARNETHON, M.; DE SIMONE, G.; FERGUSON, T.B.; FLEGAL, K.; FORD, E.; FURIE, K.; GO, A.; GREENLUND, K.; HAASE, N.; HAILPERN, S.; HO, M.; HOWARD, V.; KISSELA, B.; KITTNER, S.; LACKLAND, D.; LISABETH, L.; MARELLI, A.; McDERMOTT, M.; MEIGS, J.; MOZAFFARIAN, D.; NICHOL, G.; O’DONNELL, C.; ROGER, V.; ROSAMOND, W.; SACCO, R.; SORLIE, P.; STAFFORD, R.; STEINBERGER, J.; THOMT.; WASSERTHIEL-SMOLLER, S.; WONG, N.; WYLIE-ROSETT, J.; HONG, Y.. Heart disease and stroke statistics-2009 update: a report from the American Heart Association Statistics Committee and Stroke Statistics Subcommittee. Circulation, Dallas, v.119, n.3, p.e21-181, 2009.

LOHMAN, T.G.; ROCHE, A.F; MARTORELL, R. Antropometric standardization reference manual. Champaign: Human Kinetics, 1988.

LOOS, R.J. Recent progress in the genetics of common obesity. British Journal of Clinical Pharmacology, London, v.68, n.6, p.811-29, 2009.

LOPEZ-BERMEJO, A.; PETRY, C.J.; DIAZ, M.; SEBASTIANI, G.; DE ZEGHER, F.; DUNGER, D.B.; IBANEZ, L. The association between the FTO gene and fat mass in humans develops by the postnatal age of two weeks. Journal of Clinical Endocrinology \& Metabolism, Chevy Chase, v.93, n.4, p.1501-5, 2008.

MARTI, A.; MORENO-ALIAGA, M.J.; HEBEBRAND, J.; MARTINEZ, J.A. Genes, lifestyles and obesity. International Journal of Obesity and Related Metabolic Disorders, London, v.28, p.S29-36, 2004. Supplement 3.

MONTEIRO, A.B.; FERNANDES FILHO, J. Análise da composição corporal: uma revisão de métodos. Revista Brasileira de Cineantropometria e Desempenho Humano, Florianópolis, v.4, n.1, p.80-92, 2002.

PÉRUSSE, L.; DESPRES, J.P.; LEMIEUX, S.; RICE, T.; RAO, D.C.; BOUCHARD, C. Familial aggregation of abdominal visceral fat level: results from the Quebec family study. Metabolism: Clinical and Experimental, New York, v.45, n.3, p.378-82, 1996. 
PÉRUSSE, L.; RICE, T.; PROVINCE, M.A.; GAGNON, J.; LEON, A.S.; SKINNER, J.S.; WILMORE, J.H.; RAO, D.C.; BOUCHARD, C. Familial aggregation of amount and distribution of subcutaneous fat and their responses to exercise training in the HERITAGE family study. Obesity Research, Silverv Spring, v.8, n.2, p.140-50, 2000.

PIETROBELLI, A.; RUBIANO, F; ST-ONGE, M.P.; HEYMSFIELD, S.B. New bioimpedance analysis system: improved phenotyping with whole-body analysis. European Journal of Clinical Nutrition, London, v.58, n.11, p.1479-84, 2004. RANKINEN, T.; ZUBERI, A.; CHAGNON, Y.C.; WEISNAGEL, S.J.; ARGYROPOULOS, G.; WALTS, B.; PÉRUSSE, L.; BOUCHARD, C.. The human obesity gene map: the 2005 update. Obesity, Silver Spring, v. 14, n.4, p.529-644, 2006. RICE, T.; DESPRES, J.P.; DAW, E.W.; GAGNON, J.; BORECKI, I.B.; PÉRUSSE, L.; LEON, A.S.; SKINNER, J.S.; WILMORE, J.H.; RAO, D.C.; BOUCHARD, C. Familial resemblance for abdominal visceral fat: the HERITAGE family study. International Journal of Obesity and Related Metabolic Disorders, London, v.21, n.11, p.1024-31, 1997. RICE, T.K.; BORECKI, I.B. Familial resemblance. In: RAO, D.C.; PROVINCE, M.A. Genetic dissection of complex traits. San Diego: Academic Press, 2001.

SHEN, W.; PUNYANITYA, M.; SILVA, A.M.; CHEN, J.; GALLAGHER, D.; SARDINHA, L.B.; ALLISON, D.B.; HEYMSFIELD, S.B. Sexual dimorphism of adipose tissue distribution across the lifespan: a cross-sectional whole-body magnetic resonance imaging study. Nutrition \& Metabolism, London, v.6, p.17, 2009.

WALLEY, A.J.; BLAKEMORE, A.I.; FROGUEL, P. Genetics of obesity and the prediction of risk for health. Human Molecular Genetics, Oxford, v.15, n.2, p.R124-130, 2006. Special number.

WU, C.H.; HESHKA, S.; WANG, J.; PIERSON JUNIOR, R.N.; HEYMSFIELD, S.B.; LAFERRERE, B.; WANG, Z.; ALBU, J.B.; PI-SUNYER, X.; GALLAGHER, D. Truncal fat in relation to total body fat: influences of age, sex, ethnicity and fatness. International Journal of Obesity, London, v.31, n.9, p.1384-91, 2007.

\section{Agradecimentos}

Trabalho financiado pela Fundação para a Ciência e Tecnologia (FCT) de Portugal com referência PTDC/ DES/67569/2006. 